\title{
Advances in the diagnosis and treatment of benign paroxysmal positional vertigo (Review)
}

\author{
HENGYONG TANG ${ }^{1}$ and WEI LI ${ }^{2}$ \\ ${ }^{1}$ Department of Neurology, Fengcheng Hospital in Fengxian District; \\ ${ }^{2}$ Department of Neurology, Shanghai Ninth People's Hospital, Shanghai, P.R. China
}

Received January 30, 2017; Accepted May 19, 2017

DOI: $10.3892 /$ etm.2017.4837

\begin{abstract}
Benign paroxysmal positional vertigo (BPPV) (otolith disease) is the most common neurological and position change related vertigo, accounting for $17-20 \%$ of peripheral vertigo. BPPV occur in the elderly. The high incidence age for BPPC was 50 to 70 years and mostly in female. According to the different parts of the lesions, it is divided into anterior canal BPPV (AC-BPPV), posterior canal BPPV (PC-BPPV), horizontal canal BPPV (HC-BPPV). Studies have shown that the incidence of PC-BPPV was $86.36 \%$, the incidence of HC-BPPV was $11.37 \%$, the incidence rate of AC-BPPV was $2.27 \%$. Treatment for PC-BPPV includes manual reduction, drug treatment, psychological treatment, and surgical treatment. Repositioning is the preferred method for the treatment of PC-BPPV with high effective rate and low risk. The mechanism is through the different changes of head position to make the otolith back to utricle. Many manual reduction methods have been reported in clinical treatment of PC-BPPV. With the increasing emphasis on the BPPV, a variety of new methods are being developed and designed.
\end{abstract}

\section{Contents}

1. Introduction

2. BPPV diagnosis

3. Manual reduction of BPPV

4. Other treatments for BPPV

\section{Introduction}

Benign paroxysmal positional vertigo (BPPV) is a vertigo syndromeoccurring due to posturalchanges and is characterized

Correspondence to: Dr Wei Li, Department of Neurology, Shanghai Ninth People's Hospital, 639 Zhizaoju Road, Huangpu, Shanghai 200011, P.R. China

E-mail: rxigtu526@163.com

Key words: benign paroxysmal positional vertigo, repositioning, utricle, peripheral vertigo, otolith disease by nystagmus. BPPV was first described as vertigo caused by changes in the position of the head (1). Barany, the founder of Hungarian ear neurology, reported a female patient, aged 27 years, with vertigo which was associated with changes in the position of the head. Her vertigo tended to occur during the processes of getting up, lying down, and turning over, with transient symptoms lasting $<1 \mathrm{~min}$, and was repeatable. These symptoms were attributed to the otolithic membrane (2). Dix and Hallpike first proposed this diagnosis (3) after summarizing a large number of diseases with the same symptoms and finding a certain incubation period (mostly $<1 \mathrm{~min})$ prior to symptom onset. They also noted that the side with the ear facing downward was the affected side when vertigo occurred, that nystagmus was transient, rotary, geotropic, and apogeotropic, that nystagmus was induced again when the head rotated to its original position despite the movement being in the opposite direction (reversibility), and that nystagmus was attenuated (fatigue) after repeated position change stimulations. In 1985, McClure first reported seven BPPV patients with horizontal nystagmus induced by changing positions (4). Baloh et al (1993) (5) and Lempert (1994) (6) described the clinical characteristics of horizontal canal BPPV (HC-BPPV), and found that the inducing position and type of nystagmus for HC-BPPV were different than that of the previously reported posterior canal BPPV (PC-BPPV). Nystagmus was induced horizontally rather than via the previously reported rotary or apogeotropic motions, and corresponding reduction treatments were used (7). In 1994, Brandt first reported the clinical manifestation of PC-BPPV. Three years later, Herdman (8) found that some patients may experience vertigo and nystagmus when the position of head was changed, but the characteristics of nystagmus were different from previously reported cases: the nystagmus was induced by vertical, geotropic, and rotary movements, indicating that this was a new type of positional vertigo.

\section{BPPV diagnosis}

Otolith disease in the posterior semicircular canal typically occurs during the process of getting up or lying down (9). In 1952, Dix and Hallpike summarized the characteristics of vertigo and nystagmus in patients with PC-BPPV and developed the Dix-Hallpike test (3) (also named the Nylen-Barany test): i) the patient sits on the bed and rotates the head $45^{\circ}$, 
ii) the examiner asks the patient to change from sitting to supine position, with the head drooping to $30^{\circ}$, during which the presence of nystagmus was examined. If vertigo and nystagmus occurred after the transient incubation period, the patient was considered positive. iii) The patient is asked to return to sitting position, and vertigo and nystagmus occurred again during this process, with the nystagmus in the opposite direction and iv) the head is rotated to the other side and steps 1-3 are repeated. The degree of vertigo and the type and intensity of nystagmus was compared to determine whether the condition was PC-BPPV or anterior canal BPPV (AC-BPPV), and to identify the affected side. When significant vertigo and apogeotropic, rotary, and geotropic nystagmus occurred after a head $45^{\circ}$ rotation to the right, the condition was considered right $\mathrm{PC}-\mathrm{BPPV}$, and when symptoms occurred after a $45^{\circ}$ rotation to the left, the condition was considered left PC-BPPV. When significant vertigo and geotropic, rotary, and geotropic nystagmus occurred after the head rotated to both sides, the condition was considered bilateral PC-BPPV. Bo et al showed that when the patients with PC-BPPV underwent the Dix-Hallpike test, nystagmus was found in the direction of the affected side when the head was hanging in the supine position and in the direction of the healthy side when the patient was in sitting position; this phenomenon was noted as the interconvertibility of nystagmus (10). For patients with cervical spondylosis or cervical injury, the neck should not be stretched for too long, rendering the Dix-Hallpike test inappropriate. In such cases, the side-lying test, designed by Cohen (11), could be used. i) The patient sits on the bed with the head rotated to the non-testing side at a $45^{\circ}$ angle. ii) While maintaining head position, the patient lies on the side to be tested, with the tested-side shoulder on the bed. Presence of vertigo and nystagmus were observed. iii) While maintaining head position, the patient then returns to a sitting position while indications of vertigo and nystagmus were observed in order to diagnose PC-BPPV and SC-BPPV. Xie also designed a test for limited cervical spine motion called the modified lateral decubitus position test (12). In this test, i) the subject wears cervical gear and sits in the middle of the bed with maintained head and neck position, ii) the body is then rotated to the left at a $45^{\circ}$ angle while maintaining head position, and the patient is laid on the right side rapidly for right posterior semicircular canal examination and iii) after returing to a sitting position and resting for $1 \mathrm{~min}$, the procedure is repeated for investigation of the left posterior semicircular canal.

Otolith disorders in the horizontal semicircular canal were usually induced by head rotation and turning over on the bed. The most commonly used diagnosis was the supine roll test (also called the Pagnini-McClure maneuver), which is the gold standard for the diagnosis of HC-BPPV (13). The procedure is as follows: i) the patient is placed in a sitting position, ii) the patient moves rapidly from a sitting to supine position, iii) the head is rotated $90^{\circ}$ to one side and nystagmus in both eyes is examined and iv) the head is rotated $90^{\circ}$ to the other side and the presence of vertigo and nystagmus are examined. This method could induce two forms of nystagmus representing different types of HC-BPPV (14): when the head was rotated $90^{\circ}$ to the right and significant vertigo occurred/horizontal and geotropic nystagmus was induced, this was diagnosed as right horizontal canal otolith disorder. Otherwise, the condition was diagnosed as left horizontal canal otolith disorder. The etiology of both disorders is that the semicircular canal was affected. However, if the etiology was a lesion in the cupula cristae ampullaris, horizontal and apogeotropic nystagmus would be present, and the affected side was the side where no significant nystagmus occurred. This procedure should be prohibited for patients having short necks with poor mobility, morbid obesity, and severe kyphosis (15). Mei developed a modified supine roll test for these patients (16) with the following procedure: with the patient in supine position, the head was blocked up at an angle of $30^{\circ}$. All other steps were identical to the conventional supine roll test (16). Xie diagnosed patients with poor mobility using a modified supine positioning test (12): the patient wore a cervical and the head and neck positions were maintained. Both the head and the body were rotated $90^{\circ}$ to one side, any occurrence of vertigo and nystagmus were determined, and the patient was returned to the sitting position. After $2 \mathrm{~min}$, both the head and body were rotated $90^{\circ}$ to the other side and any occurrence of vertigo and horizontal nystagmus were determined. The affected semicircular canal was determined based on the occurrence and type of nystagmus.

Apart from the commonly used supine roll test for HC-BPPV, Rahko and Kotti reported a walk-rotate-walk test (17), where the patient is rotated $180^{\circ}$ with the heel serving as the axis during fast walking, then returned to the original position at the same speed. This cycle was repeated three times without any pause. Astasia, manual balance correction, and the need for pauses were observed during fast rotation. HC-BPPV could be diagnosed when these actions were observed repeatedly, and the side to which the patient rotated to was considered the affected side. Nuti et al used the head yaw test to diagnose HC-BPPV. The patient started in a supine position and rapidly rotated their head $90^{\circ}$ to the side of the tested ear. As the rapid downward movement leads to nystagmus, called geotropic nystagmus, conversely, called apogeotropic nystagmus, Nuti et al developed the seated supine positioning test (18), where the patient's head was held at $30^{\circ}$ while the patient changed from sitting to supine position, potentially inducing seated supine positioning nystagmus. HC-BPPV patients with geotropic nystagmus rotated to the healthy side, while the HC-BPPV patients with apogeotropic nystagmus rotated to the affected side. Choung et al developed the bow and lean test to diagnose HC-BPPV (19): i) the HC-BPPV was bile duct stone HC-BPPV or crest stone HC-BPPV, ii) the patient is in a sitting position and bows their head greater than $45^{\circ}$, and the direction of nystagmus was observed (specific for bile duct stone HC-BPPV) and iii) the patient then throws their head back greater than $45^{\circ}$ and the direction of nystagmus was observed (specific for crest stone HC-BPPV). When patients with bile duct stone HC-BPPV bowed their heads, nystagmus was induced in the direction of the affected semicircular canal. When patients with crest stone HC-BPPV threw their heads back, nystagmus was induced in the direction of the affected semicircular canal.

AC-BPPV was mainly induced during the process of getting up and lying down, and the diagnosis method was the same as with PC-BPPV. If vertigo and geotropic nystagmus occurred during the position-induced test, the diagnosis was AC-BPPV. If apogeotropic nystagmus occurred, it was contralateral AC-BPPV, and if geotropic nystagmus occurred, it was 


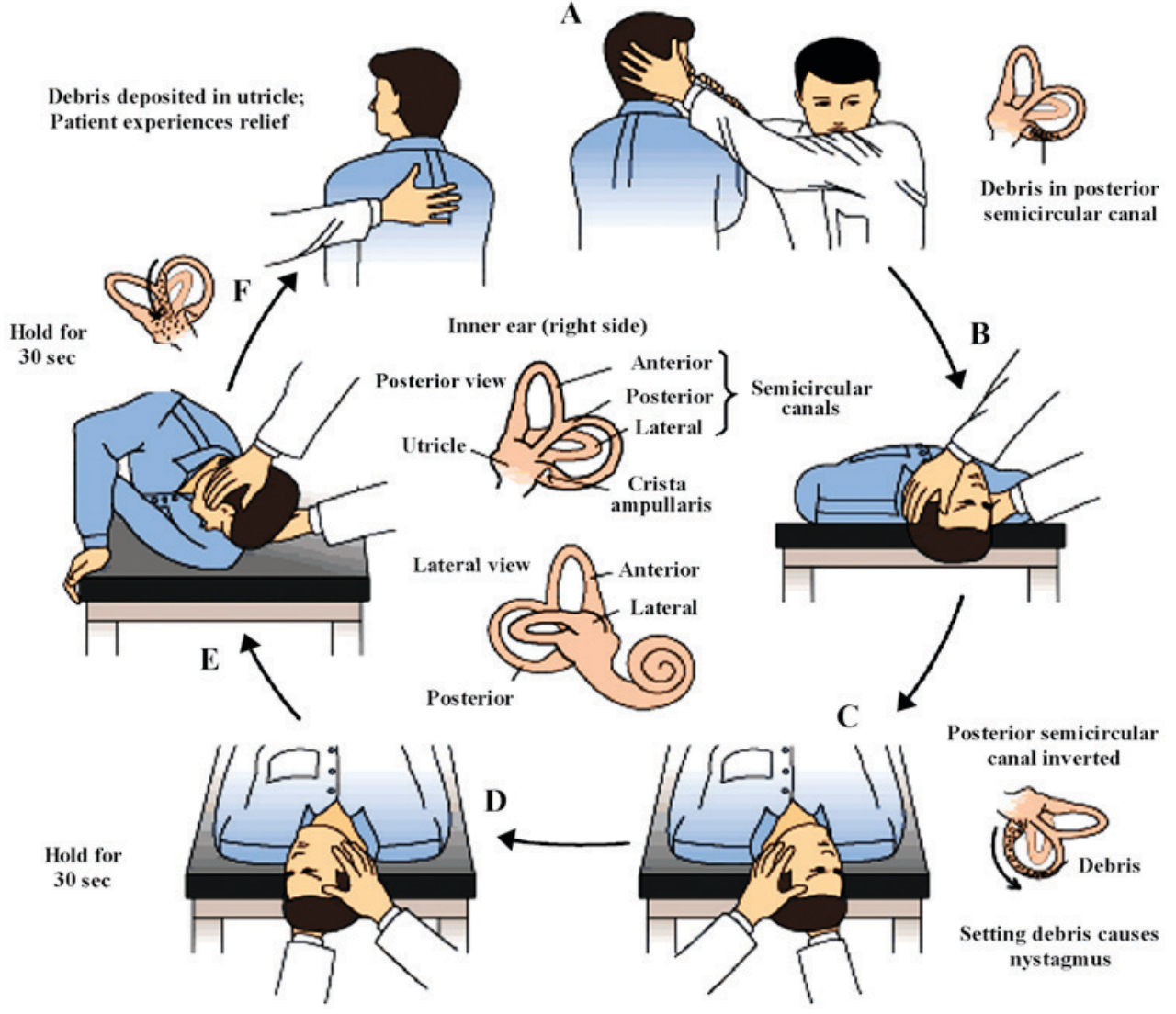

Figure 1. Evaluation of nystagmus after each procedure. The subsequent procedure was performed $1 \mathrm{~min}$ after resolution of nystagmus.

homolateral AC-BPPV. In addition, Rahko developed another diagnosis method for AC-BPPV (20): the patient, standing upright, closes their eyes and stoops down to a $60^{\circ}$ angle, then rapidly stands upright again. If the patient toppled over to any side, they would be diagnosed with AC-BPPV. The shortcoming of these two methods was the presence of false positive diagnoses. Meng reported that the self-made SRM-IV system was sensitive to AC-BPPV, and speculated that the actual morbidity of AC-BPPV was much higher than presented in previous statistics due to missed diagnoses in conventional detection (21).

Recently, examination of vestibular function has been extensively applied for the diagnosis of BPPV. For example, the vestibular autorotation test can determine the function of both horizontal and vertical semicircular canals (22). Imaging has also been increasingly applied in the diagnosis of BPPV. Li reported that positive cranial MRI in BPPV patients showed affected semicircular canal and ear bones, as well as otolith detachment (23). Heavily T2-weighted magnetic resonance imaging of the inner ear can clearly demonstrate the internal acoustical meatus structure and allow full observation of the inner ear (24). Yan performed heavily T2-weighted magnetic resonance imaging of the ossa temporale in $48 \mathrm{BPPV}$ patients and found that magnetic resonance imaging of the inner ear could provide imaging evidence for the diagnosis of BPPV (25).

\section{Manual reduction of BPPV}

In 1980, Brandt and Daroff developed an exercise for the treatment of otolith disease (26): the patient starts in a sitting position, changes rapidly to a right supine position with the head rotated at a $45^{\circ}$ angle to the left. Thirty seconds following the resolution of the resultant vertigo, the patient would sit rapidly and stare forward for another $30 \mathrm{sec}$, then change rapidly to a left supine position with the head rotated at a $45^{\circ}$ angle to the right. This would be repeated 20 times, twice daily, 1-2 times per week, and could be gradually stopped after symptom remission. In 1988, Semont et al developed the modified liberatory maneuver based on the theory of crest otolith (27). The clinical significance of the modified liberatory maneuver was that only a single positioning exercise was required to achieve a cure. The procedure is as follows: i) the patient, in a sitting position, rotates their head to the healthy side at $45^{\circ}$, ii) the patient then switches rapidly to a lateral supine position while maintaining the position of their head, iii) the patient then switches from the affected side to the healthy side while again maintaining head direction (the apex nasi contacts the bed) and iv) the patient recovers to a sitting position with the head tilted forward at $20^{\circ}$. Nystagmus was evaluated after each procedure, and the subsequent procedure was performed 1 min after resolution of nystagmus (Fig. 1). van der Scheer-Horst et al performed a retrospective review of the effectiveness of Brandt-Daroff vestibular rehabilitation and manual reduction, and found that the onset of manual reduction was earlier than vestibular rehabilitation, yet the combination of vestibular rehabilitation and manual reduction was beneficial for the recovery of patients (28). Patients with limited cervical spine motion could use the modified Semont procedure (29): i) the patient wears a cervical gear while sitting on the front of the bed, then while maintaining 


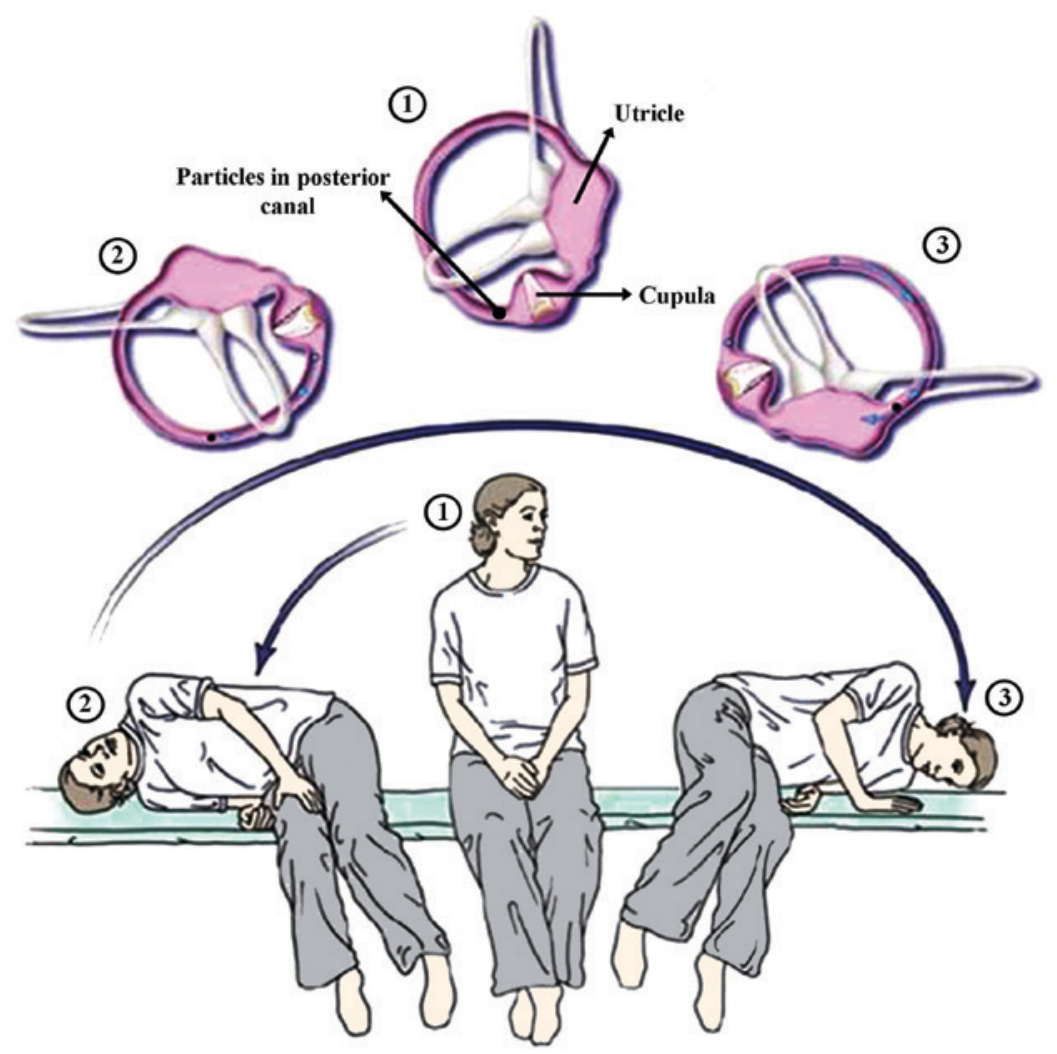

Figure 2. Procedures of Epley reduction for treatment of PC-BPPV. PC-BPPV, posterior canal benign paroxysmal positional vertigo.

head and neck position, rotates the body $45^{\circ}$ to the left in order to treat the right posterior semicircular canal, ii) the patient changes rapidly to a right supine position (the same with the modified lateral supine position), the back of the head contacts the bed, and the apex nasi faces upward, iii) after resolution of vertigo and nystagmus, the patient changes from a right supine to a left supine position with the rear arm as the pivot point, the forehead and apex nasi contacting the bed, and the back of the head facing upward and iv) the patient sits up. The treatment for the left posterior semicircular canal was the same as that for the right posterior semicircular canal, except for reversed directions.

The most classical treatment for PC-BPPV is the Epley reduction, which was developed by Epley based on the hypothesis of otolith in the semicircular canal (30): i) the patient starts in a sitting position with their head tilted to the affected side at a $45^{\circ}$ angle, ii) the patient changes from a sitting to a supine position with the head beneath the bed at an angle of $45^{\circ}$, iii) while maintaining the positioning of the body, the head was then rotated to the healthy side at a $45^{\circ}$ angle, iv) both the body and the head then rotated to the healthy side at $90^{\circ}$ angles, v) the body was then shifted to a supine position towards the healthy side, with an angle of $135^{\circ}$ between the head and the bed and vi) the patient then sat and tilted their head forward at a $20^{\circ}$ angle. Each position was maintained for $1 \mathrm{~min}$. Rotation was stopped upon vertigo and continued $30 \mathrm{sec}$ after resolution of nystagmus (Fig. 2). Epley's therapy was highly effective: success rates were found to be $90.7 \%$ after one conductance of an Epley reduction and $96.0 \%$ after two conductances. However, this therapy was not appropriate for patients with limited cervical spine motion, who could use the Semont procedure (31). Anagnostou et al treated 51 PC-BPPV patients each with the Epley procedure or the Semont procedure and found that the successful reduction rate was not significantly different between these two therapies: $7.8 \%$ of patients had HC-BPPV after treatment by the Epley procedure, while no patient had HC-BPPV after treatment by the Semon procedure (32). Yang demonstrated a modified 3 -step procedure which was not limited to a specific treatment platform and could be performed on regular bed if a hard and $15-\mathrm{cm}$ high pillow was put on the bed (33). This procedure was very convenient for clinical treatment. The reduction could be performed as three steps: i) the patient, in a sitting position and head rotated to the affected side at a $45^{\circ}$ angle, lies down rapidly to create shoulder contact with the pillow while the head remains above the pillow facing downward at an angle of $30^{\circ}$, ii) the patient then rotates their head to the healthy side and rotates over into the prone position, then tilts their head back at an angle of $30^{\circ}$ and iii) the patient reverts to sitting position with the head tilted forward at an angle of $30^{\circ}$.

In 1990, Baloh et al (34) and Lempert (6) reported that most commonly used treatment for HC-BPPV reduction was the barbecue roll maneuver, which aimed to remove the otolith from the horizontal semicircular canal (6). i) The patient starts in the supine position with the head rotated $90^{\circ}$ to the healthy side, ii) the body then rotates $180^{\circ}$, moving the patient from the supine to the prone position without changing the position of the head, iii) the head maintains its $90^{\circ}$ rotation while the apex nasi faces downward, iv) with the head still aligned at $90^{\circ}$ towards the healthy side, the lateral face contacts the bed and v) the patient sits up again (35). The mean effective rate of $\mathrm{BRM}$ for $\mathrm{HC}-\mathrm{BPPV}$ was $75 \%$, with a reported range 
of $50-100 \%$ (36). BRM was more suitable for otolith disease or a crest otolith adhering to the cupula canal-side (CUP-C), and ineffective for a crest otolith adhering to the cupulautricle side (CUP-U) (37). Patients with obesity, cervical spondylosis, and muscle disease could not tolerate BRM due to limited positioning (38). In 1998, Gufoni et al developed a new treatment for HC-BPPV to complement BRM (39): i) the patient moves from a sitting to a lateral supine position (from CUP-C to the healthy side, from CUP-U to the affected side) while keeping the position of the head forward, ii) the head is rotated $45^{\circ}$ to the healthy side without altering the position of the body and iii) the patient resumes a sitting position with the head position unchanged. Each position was maintained for $3 \mathrm{~min}$.

The Gufoni procedure is convenient and highly effective, as well as suitable for all types of HC-BPPV (including otolith disease and crest otolith) (39). As vertigo symptoms were mild on the tilted side during reduction, the patient could tolerate the treatment). HC-BPPV patients with horizontal apogeotropic nystagmus could perform this step-by-step reduction (40-42), as Gufoni otolith detachment could transform horizontal apogeotropic nystagmus into horizontal geotropic nystagmus, at which point BRM could be performed to achieve good efficacy. Mei used a self-made otolith repositioning procedure to transform horizontal apogeotropic nystagmus into horizontal geotropic nystagmus, and also achieved good efficacy (43). Testa developed a modified Gufoni procedure to modify the turbulent flow in the ampulla during the motion of the semicircular canal so that the otolith materials were far away from crura commune. As a result, HC-BPPV was less likely to be transformed into PC-BPPV, thereby improving the efficacy from the classical Gufoni procedure (44). The Vannucchi-Asprella maneuver was another option for HC-BPPC and more convenient than BRM. However, it was limited by a requirement for successive multiple cycles to achieve good efficacy (38): i) the patient starts in supine position with the head rotated $90^{\circ}$ to the healthy side, ii) the patient sits up slowly without changing head position, iii) the head slowly moves to face forward and iv) the patient moves from sitting to supine position. This procedure should be repeated several times. This procedure was considered a modified BRM, as it placed the otolith back into the alveus communis using gravity and angular acceleration (rotation of the head in supine position) through the changing between sitting and supine positions. Vannucchi-forced prolonged positioning is generally used as a complementary therapy for BRM rather than a single therapy. Vannucchi first developed this procedure in 1994 for patients with otolith disease. This procedure required the patient to maintain supine position on the healthy side for at least $12 \mathrm{~h}$ so as to ensure the opening of the alveus communis in the horizontal semicircular canal of the affected side was always facing downward, so that the otolith could returned to the alveus communis via gravity. Head rotation in a supine position could also be used to treat this BPPV: with the patient in supine position, the head is rapidly rotated $90^{\circ}$ to the healthy side (for patients with otolith disease), then returned to the initial position slowly. The procedure was then repeated. Also, patients with crest otolith should rotate their heads to the affected side (45). The modified Epley procedure could also be used for AC-BPPV: the procedure was similar to the Epley procedure but with a slow frequency of reduction, a pause between each position longer than $1 \mathrm{~min}$, and each maneuver was performed by the patients themselves (46). The Li maneuver was usually used for the treatment of AC-BPPV: i) the patient starts in supine position with head facing $20^{\circ}$ downward and legs on both sides of the bed and ii) the patient then sits rapidly, changing from supine to prone position with the face contacting the bed. In 2009, the Yacovino procedure was first reported for the treatment of AC-BPPV (47): i) the patient starts in a sitting position with the head facing forward, ii) the patient rapidly changes to supine position with the head moving upward at a minimum of $20^{\circ}$ for $2-3 \mathrm{~min}$, iii) the head is elevated and bent forward for 2-3 min and iv) the patient returns to a sitting position and looks forward horizontally. The healthy or affected sides may not be considered. A previous study suggested that it was difficult to provide maximum stimulation to the affected side, given that it was left undetermined, possibly impacting efficacy (48). Xing suggested that the effective rate of this procedure was consistent with the modified Epley reduction, but the procedure itself was easier to perform (49).

Recently, whether position limitation was required is controversial. Some studies (50-52) and a recent meta-analysis by Mostafa et al (53) showed that the difference between limited motion and free motion after reduction was not significant. Zhang used manual reduction to treat PC-BPPV with good efficacy and no adverse reactions (54). Li used semi-supine positioning and a turning-over exercise to treat elderly BPPV patients; this procedure contained characteristic small motions and ease of operation, making it more acceptable in elderly patients than conventional reductions (55).

With the development of technology, more and more reduction machines have been implemented. The SRM-IV BPPV diagnosis and treatment system (developed in China) provided automatic, precise, quantitative, rapid diagnosis, and reduction for BPPV patients $(56,57)$ with a total efficacy of $97.02 \%(58)$, which was superior to conventional manual reduction. This system could also reduce the incidence of vomiting, pale face, and cold sweats during artificial reduction (59). The TRV otolith instrument first appeared at the 111th Annual Meeting of French ENT (60); its efficacy was higher than most manual reductions (61). $\mathrm{Xu}$ reported a novel automatic 3D chair to assist manual reduction, but the clinical efficacy of this device requires further observation (62).

\section{Other treatments for BPPV}

Prior to the application of manual reduction, drugs that improve microcirculation and inhibit the vestibule have been used as first line drugs for BPPV, and have been shown to improve efficacy (63-65). For refractory BPPV patients, manual reduction alone could not achieve good efficacy, so two surgeries could be considered: i) single neurectomy could improve the clinical symptoms of BPPV patients. In fact, this surgery can achieve good efficacy, yet some patients may be subjected to high risk of hearing loss. ii) Semicircular canal occlusion can be used to occlude the space in the crest with special material to reduce the stimulation of the hair cell sensory receptors in the ampulla. This procedure has low risk. However, surgeries may cause severe hearing loss, and thus should be considered 
carefully and should only be used when the efficacy of manual reduction and medical treatment was unsatisfactory.

\section{References}

1. van der Plas JP and Tijssen CC: Benign paroxysmal positional vertigo. Ned Tijdschr Geneeskd 142: 2669-2674, 1998 (In Dutch).

2. Bestuzheva NV, Parfenov VA and Zamergrad MV: Benign paroxysmal positional vertigo in a female with arterial hypertension and meningioma. Zh Nevrol Psikhiatr Im S S Korsakova 114: 100-104, 2014 (In Russian).

3. Dix MR and Hallpike CS: The pathology symptomatology and diagnosis of certain common disorders of the vestibular system. Proc R Soc Med 45: 341-354, 1952.

4. McClure JA: Horizontal canal BPV. J Otolaryngol 14: 30-35, 1985.

5. Baloh RW, Jacobson K and Honrubia V: Horizontal semicircular canal variant of benign positional vertigo. Neurology 43 : 2542-2549, 1993.

6. Lempert T: Horizontal benign positional vertigo. Neurology 44: 2213-2214, 1994.

7. Brandt T, Steddin S and Daroff RB: Therapy for benign paroxysmal positioning vertigo, revisited. Neurology 44 796-800, 1994

8. Herdman SJ: Advances in the treatment of vestibular disorders. Phys Ther 77: 602-618, 1997.

9. Curthoys IS and Manzari L: Otolithic disease: clinical features and the role of vestibular evoked myogenic potentials. Semin Neurol 33: 231-237, 2013.

10. Bo Z and Sun J: The characteristic of nystagmus in naked eyes and electronystagmogram of BPPV patients and the location diagnosis analysis. J Audiol Speech Pathol 20: 235-237, 2012.

11. Cohen HS: Side-lying as an alternative to the Dix-Hallpike test of the posterior canal. Otol Neurotol 25: 130-134, 2004.

12. Xie X: The diagnosis and treatment of BPPV after cervical spine surgery. Chin J EENT 14: 151-153, 2004.

13. Giannini S, Signorini L, Bonanome L, Severino M, Corpaci F and Cielo A: Benign paroxysmal positional vertigo (BPPV): it may occur after dentalimplantology. A mini topical review. Eur Rev Med Pharmacol Sci 19: 3543-3547, 2015.

14. Luis L, Costa J, Vaz Garcia F, Valls-Solé J, Brandt T and Schneider E: Spontaneous plugging of the horizontal semicircular canal with reversible canal dysfunction and recovery of vestibular evoked myogenic potentials. Otol Neurotol 34 743-747, 2013.

15. Psillas G, Vital I, Rachovitsas D, Vital V and Tsalighopoulos M: Conversion of canalolithiasis to cupulolithiasis in the course of a horizontal benign paroxysmal positional vertigo case. Am J Otolaryngol 32: 174-176, 2011.

16. Mei X: The diagnosis of horizontal canal BPPV by the modified roll test. Mod Diagn Treat 25: 1959-1961, 2014.

17. Rahko T and Kotti V: Walk-rotate-walk test identifies patients responding to Lempert's maneuver, with benign paroxysmal positional vertigo of the horizontal canal. Eur Arch Otorhinolaryngol 258: 112-115, 2001.

18. Nuti D, Vannucchi P and Pagnini P: Lateral canal BPPV: which is the affected side? Audiol Med 3: 16-20, 2005.

19. Choung YH, Shin YR, Kahng H, Park K and Choi SJ: 'Bow and lean test' to determine the affected ear of horizontal canal benign paroxysmal positional vertigo. Laryngoscope 116: 1776-1781, 2006.

20. Rahko T: The test and treatment methods of benign paroxysmal positional vertigo and an addition to the management of vertigo due to the superior vestibular canal (BPPV-SC). Clin Otolaryngol Allied Sci 27: 392-395, 2002

21. Talaat HS, Kabel AM, Khaliel LH, Abuhadied G, El-Naga HA and Talaat AS: Reduction of recurrence rate of benign paroxysmal positional vertigo by treatment of severe vitamin D deficiency. Auris Nasus Larynx 43: 237-241, 2016.

22. Jiang Z: The importance of vestibular examination in the diagnosis of vertigo. Chin Med J (Engl) 91: 32-41, 2011.

23. $\mathrm{Li} \mathrm{H}$ : The importance of inner ear MRI in the diagnosis of BPPV (85 cases). Chin J Mod Med 22: 84-86, 2012.

24. Zhang $\mathrm{Y}, \mathrm{Gao} \mathrm{G}$ and $\mathrm{Xu} \mathrm{O}$ : MRI of posterior circulation ischemia vertigo. Zhonghua Er Bi Yan Hou Tou Jing Wai Ke Za Zhi 18: 70-72, 2011.

25. Yan J: The importance of inner ear MRI in the diagnosis of BPPV. J Intern Intensive Med 19: 93-94, 2013.

26. Brandt T and Daroff RB: Physical therapy for benign paroxysmal positional vertigo. Arch Otolaryngol 106: 484-485, 1980.
27. Semont A, Freyss G and Vitte E: Curing the BPPV with a liberatory maneuver. Adv Otorhinolaryngol 42: 290-293, 1988.

28. van der Scheer-Horst ES, van Benthem PP, Bruintjes TD, van Leeuwen RB and van der Zaag-Loonen HJ: The efficacy of vestibular rehabilitation in patients with benign paroxysmal positional vertigo: a rapid review. Otolaryngol Head Neck Surg 151: 740-745, 2014.

29. Okubadejo GO, Hohl JB and Donaldson WF: Dysphonia, dysphagia, and esophageal injuries after anterior cervical spine surgery. Instr Course Lect 58: 729-736, 2009.

30. Epley JM: The canalith repositioning procedure: for treatment of benign paroxysmal positional vertigo. Otolaryngol Head Neck Surg 107: 399-404, 1992.

31. Babac S and Arsović N: Efficacy of Epley maneuver in treatment of benign paroxysmal positional vertigo of the posterior semicircular canal. Vojnosanit Pregl 69: 669-674, 2012 (In Serbian).

32. Anagnostou E, Stamboulis E and Kararizou E: Canal conversion after repositioning procedures: comparison of Semont and Epley maneuver. J Neurol 261: 866-869, 2014

33. Yang Q: The efficacy of modified 3-step manual reduction in the treatment of posterior canal HBBP. Clin J Otorhinolaryngol Head Neck 28: 1796-1798, 2014.

34. Baloh RW, Honrubia V and Jacobson K: Benign positional vertigo: clinical and oculographic features in 240 cases. Neurology 37: 371-378, 1987.

35. Kim JS, Oh SY, Lee SH, Kang JH, Kim DU, Jeong SH, Choi KD, Moon IS, Kim BK and Kim HJ: Randomized clinical trial for geotropic horizontal canal benign paroxysmal positional vertigo. Neurology 79: 700-707, 2012

36. Mandalà M, Pepponi E, Santoro GP, Cambi J, Casani A, Faralli M, Giannoni B, Gufoni M, Marcelli V, Trabalzini F, et al: Double-blind randomized trial on the efficacy of the Gufoni maneuver for treatment of lateral canal BPPV. Laryngoscope 123: $1782-1786,2013$

37. Riga M, Korres S, Korres G and Danielides V: Apogeotropic variant of lateral semicircular canal benign paroxysmal positional vertigo: is there a correlation between clinical findings, underlying pathophysiologic mechanisms and the effectiveness of repositioning maneuvers? Otol Neurotol 34: 1155-1164, 2013.

38. Korres S, Riga MG, Xenellis J, Korres GS and Danielides V: Treatment of the horizontal semicircular canal canalithiasis: pros and cons of the repositioning maneuvers in a clinical study and critical review of the literature. Otol Neurotol 32: 1302-1308, 2011.

39. Gufoni M, Mastrosimone L and Di Nasso F: Repositioning maneuver in benign paroxysmal vertigo of horizontal semicircular canal. Acta Otorhinolaryngol Ital 18: 363-367, 1998 (In Italian).

40. Qi Z, Zhao F and Zhuang J: The experience of treatment and treatment of horizontal canal BPPV with apogeotropie nystagmus. Chin J EENT 14: 142-144, 2014.

41. Chen Y: The treatment of horizontal canal otolith disease and crest otolith in the ampulla by sequential method. Clin J Otorhinolaryngol Head Neck 28: 10-12, 2014.

42. Ren Y: The efficacy of barbecue roll maneuver for horizontal BPPV. Clin J Otorhinolaryngol Head Neck 20: 356-357, 2014

43. Mei X: The diagnosis and manual reduction of horizontal apogeotropie BPPV. Clin J Otorhinolaryngol Head Neck 28: 15-17, 2014

44. Testa D, Castaldo G, De Santis C, Trusio A and Motta G: Treatment of horizontal canal benign paroxysmal positional vertigo: a new rehabilitation technique. Sci World J 2012: 160475, 2012.

45. Vannucchi P and Pecci R: Pathophysiology of lateral semicircular canal paroxysmal positional vertigo. J Vestib Res 20 433-438, 2010.

46. Parnes LS, Agrawal SK and Atlas J: Diagnosis and management of benign paroxysmal positional vertigo (BPPV). CMAJ 169: 681-693, 2003

47. Chen D, Xiong S and Cui Y: Treatment of anterior canal benignparoxysmal positional vertigo by Yacovino repositioning maneuver. Lin Chung Er Bi Yan Hou Tou Jing Wai Ke Za Zhi 29: $17-19,2015$

48. Das S and Rea PA: Bilateral posterior semi-circular canal obliteration surgery for refractory benign paroxysmal positional vertigo (BPPV) in three patients. Clin Otolaryngol 42: 480-483, 2017.

49. Xing D: The efficacy of suspended head reduction for posterior canal BPPV. J Audiol Speech Pathol 22: 524-526, 2014.

50. De Stefano A, Dispenza F, Citraro L, Petrucci AG, Di Giovanni P, Kulamarva G, Mathur N and Croce A: Are postural restrictions necessary for management of posterior canal benign paroxysmal positional vertigo? Ann Otol Rhinol Laryngol 120: 460-464, 2011. 
51. Papacharalampous GX, Vlastarakos PV, Kotsis GP, Davilis D and Manolopoulos L: The role of postural restrictions after BPPV treatment: real effect on successful treatment and BPPV's recurrence rates. Int J Otolaryngol 2012: 932847, 2012.

52. Balikci HH and Ozbay I: Effects of postural restriction after modified Epley maneuver on recurrence of benign paroxysmal positional vertigo. Auris Nasus Larynx 41: 428-431, 2014.

53. Mostafa BE, Youssef TA and Hamad AS: The necessity of post-maneuver postural restriction in treating benign paroxysmal positional vertigo: a meta-analytic study. Eur Arch Otorhinolaryngol 270: 849-852, 2013.

54. Zhang F: The efficacy of a new modified manual reduction for pediatric BPPV. Chin Med 9: 124-125, 2012.

55. Li Z: Sixty cases of semi-supine turning-over exercise in the treatment of elderly BPPV patients. Southwest J Med 24: 751-753, 2012.

56. Shan X, Ma L and Peng X: Clinical study of the treatment and diagnosis of BPPV by BPPV system. Chin J Stroke 8: 340-345, 2013.

57. Sun Q: The feasibility analysis of SRM-IV in speed ladder test. Chin J Otol 12: 467-469, 2014
58. Yuan H: The efficacy of SRM-IV vestibular function diagnosis and treatment system for BPPV. J Audiol Speech Pathol 22: 308-310, 2014

59. Wang J: Clinical efficacy of SRM-IV vestibular function diagnosis and treatment system, Air Force. J Med 28: 138-140, 2012.

60. Richard-Vitton T and Viirre E: Unsteadiness and drunkenness sensations as a new sub-type of BPPV. Rev Laryngol Otol Rhinol (Bord) 132: 75-80, 2011.

61. Tian Y, Zhang T and Jiang X: Diagnosis and treatment of 318 benign paroxysmal positional vertigo cases. Lin Chung Er Bi Yan Hou Tou Jing Wai Ke Za Zhi 26: 13-15, 2012 (In Chinese).

62. Xu F: The design and development of a chair for antumatic reduction of otolith. Chin J Med Instr 37: 103-106, 2013.

63. Zhang H, Geng M and Yan B: Comparative analysis of simple manual reduction and concomitant medication in the treatment of HBBP. Clin J Otorhinolaryngol Head Neck 26: 750-752, 2012.

64. Pan J: Clinical observation of combined manual reduction in the treatment of HBBP. Clin Ration Drug Use 7: 108, 2014.

65. Guneri EA and Kustutan O: The effects of betahistine in addition to epley maneuver in posterior canal benign paroxysmal positional vertigo. Otolaryngol Head Neck Surg 146: 104-108, 2012. 